\title{
Intratherapeutic Biokinetic Measurements, Dosimetry Parameter Estimates, and Monitoring of Treatment Efficacy Using Cerenkov Luminescence Imaging in Preclinical Radionuclide Therapy
}

\author{
Oskar Vilhelmsson Timmermand ${ }^{1}$, Thuy A. Tran ${ }^{2}$, Sven-Erik Strand ${ }^{1,2}$, and Johan Axelsson ${ }^{3}$ \\ ${ }^{1}$ Department of Medical Radiation Physics, Lund University, Lund, Sweden; ${ }^{2}$ Lund University Bioimaging Center, Lund University, \\ Lund, Sweden; and ${ }^{3}$ Division of Atomic Physics, Department of Physics, Lund University, Lund, Sweden
}

\begin{abstract}
In recent years, there has been increasing interest in noninvasive Cerenkov luminescence imaging (CLI) of in vivo radionuclide distribution in small animals, a method proven to be a highthroughput modality for confirmation of tracer uptake. 11B6 is an $\operatorname{lgG}_{1}$ monoclonal antibody that is specific to free human kallikreinrelated peptidase 2 , an antigen abundant in malignant prostatic tissue. Free human kallikrein-related peptidase 2 was targeted in prostate cancer xenografts using ${ }^{177}$ Lu-labeled $11 \mathrm{~B} 6$ in either murine or humanized forms for radionuclide therapy. In this setting, CLI was investigated as a tool for providing parameters of dosimetric importance during radionuclide therapy. First, longitudinal imaging of biokinetics using CLI and SPECT was compared. Second, the CLI signal was correlated to quantitative ex vivo tumor activity measurements. Finally, CLI was used to monitor the radionuclide treatment, and the integrated CLI radiance was found to correlate well with subject-specific tumor volume reduction. Methods: 11B6 was radiolabeled with ${ }^{177} \mathrm{Lu}$ through the $\mathrm{CHX}-\mathrm{A}^{\prime \prime}$-DTPA chelator. In vivo CLI and SPECT imaging of ${ }^{177}$ Lu-DTPA-11B6 uptake was performed on NMRI and BALB/c nude mice with subcutaneous LNCaP xenografts up to $14 \mathrm{~d}$ after injection. Tumor size was measured to assess response to radionuclide therapy. Results: CLI correlated well with SPECT imaging and could be applied up to $14 \mathrm{~d}$ after injection of $20 \mathrm{MBq}$ with the specific tracer used. Through integration of the CLI radiance as a function of time, a dose metric for the tumors could be formed that correlated exponentially with tumor volume reduction. Conclusion: CLI provided valuable intratherapeutic biokinetic measurements for treatment monitoring and could be used as a tool for subject-specific absorbed dose estimation.
\end{abstract}

Key Words: Cerenkov luminescence imaging; radionuclide therapy; prostate cancer; human kallikrein related peptidase 2; human kallikrein gene family; ${ }^{177}$ Lu-DTPA-11B6; molecular imaging; dosimetry; optical imaging

J Nucl Med 2015; 56:444-449

DOI: 10.2967/jnumed.114.148544

Received Sep. 15, 2014; revision accepted Jan. 12, 2015.

For correspondence or reprints contact: Johan Axelsson, Department of Physics, Division of Atomic Physics, Lund University, Box 118, SE-221 00 Lund, Sweden.

E-mail: Johan.Axelsson@fysik.Ith.se

Published online Feb. 5, 2015.

COPYRIGHT (c) 2015 by the Society of Nuclear Medicine and Molecular Imaging, Inc.
$\mathbf{P}$ therapy are often conducted on small-animal models. Usually, the biokinetics are evaluated with ex vivo measurements of groups of animals at different time points, followed by a dosimetry calculation relying on the biokinetics data (1). This evaluation is usually done before treatment to obtain a theoretic therapeutic window to guide the researchers (2). The method suffers from the lack of real intratherapy data for each animal and, hence, the lack of an individually estimated absorbed dose to each treated tumor xenograft. As for humans, the biokinetics in preclinical trials differ between individuals, especially during therapy since the tumor volume is most likely to change within the treatment time (3). Thus, for better accuracy of the dosimetry and for correlation with biologic effect, the intratherapy biokinetics should be estimated for each individual animal. The most common tools today for such an evaluation are PET or SPECT imaging. However, these techniques are time- and labor-consuming for large cohorts of animals.

Recently, Cerenkov luminescence imaging (CLI) was introduced as a new modality to image radionuclides administered to small animals (4-6). Cerenkov emission occurs from charged particles in tissue whenever the kinetic energy of a $\beta$ particle exceeds $219 \mathrm{keV}$, assuming a tissue refractive index of 1.4. The $\beta$ radiation from many radionuclides used for PET and SPECT, and many $\beta$-emitting radionuclides used for therapy, fulfill this criterion. CLI and PET correlation has been extensively studied. Ruggiero et al. showed good correlation between CLI and PET when applying ${ }^{89} \mathrm{Zr}$-J591 to image prostate cancer xenografts for $96 \mathrm{~h}$ (7). Holland et al. also showed that CLI and PET agreed well when imaging breast cancer xenografts using ${ }^{89} \mathrm{Zr}$-trastuzumab (8). Xu et al. used CLI and PET to monitor drug efficacy in lung cancer or prostate cancer xenografts for which ${ }^{18} \mathrm{~F}$-FDG or ${ }^{18} \mathrm{~F}$-fluorothymidine was applied during tumor treatment using bevacizumab. They reported a decrease in signal for lung cancer xenografts $3 \mathrm{~d}$ after treatment initiation, demonstrating the potential of CLI as a tool for treatment monitoring (9). The ability to image ${ }^{177} \mathrm{Lu}$ using CLI in vivo was demonstrated by Price et al., who imaged a SKOV-3 ovarian cancer xenograft $24 \mathrm{~h}$ after injection of ${ }^{177} \mathrm{Lu}$-octapa-trastuzumab (10). Balkin et al. showed the use of CLI as a complement to traditional biodistribution studies in which ${ }^{177}$ Lu-DOTA-30F11 or ${ }^{90}$ Y-DOTA-30F11 was imaged up to $3 \mathrm{~d}$ after injection in a leukemia animal model (11).

We propose a method for faster evaluation of preclinical radionuclide therapy. To test this method, we relied on Cerenkov emission from a weak Cerenkov emitter, ${ }^{177} \mathrm{Lu}$. In the present study, CLI was investigated as a tool for providing subject-specific 
parameters of dosimetric importance in relation to radionuclide therapy. More specifically, whether the tumor uptake could be monitored longitudinally after a single administration of the radiopharmaceutical was investigated. In addition, the CLI signal as a function of time was numerically integrated, that is, area under the curve (AUC), and compared with the tumor volume change in animals undergoing radionuclide therapy. On the basis of the results presented here, we suggest the use of CLI as a tool for subject-specific preclinical radionuclide therapy monitoring.

\section{MATERIALS AND METHODS}

\section{Radionuclide, Conjugation, and Radiolabeling}

${ }^{177} \mathrm{Lu}$ has a half-life of $6.65 \mathrm{~d}$ and decays predominantly by emission of $\beta^{-}$with maximum and average energy of 497 and $149 \mathrm{keV}$, respectively. ${ }^{177} \mathrm{Lu}$ also emits $\gamma$ photons, enabling SPECT imaging. Gamma photons of ${ }^{177} \mathrm{Lu}$, with the energies $113 \mathrm{keV}$ (6.17\%) and 208 $\mathrm{keV}(10.4 \%)$, were used for SPECT imaging (12).

The $\mathrm{IgG}_{1}$ murine or humanized monoclonal antibody 11B6 (provided by the University of Turku and Innovagen $\mathrm{AB}$, respectively) was conjugated with the chelator CHX-A" -DTPA (Macrocyclics) according to the supplemental methods (available at http://jnm.snmjournals.org).

For radiolabeling, typically $150 \mu \mathrm{L}$ of conjugated 11B6 $(\sim 1 \mu \mathrm{g} / \mu \mathrm{L}$ in $0.2 \mathrm{M}$ in ammonium acetate buffer, $\mathrm{pH} 5.5$ ) was mixed with a predetermined amount $(\sim 150-200 \mathrm{MBq})$ of ${ }^{177} \mathrm{LuCl}_{3}$ (IDB Petten BV). Further details are provided in the supplemental methods.

\section{Cell Line and Animals}

LNCaP (human kallikrein-related peptidase 2-positive) cells were purchased from American Type Culture Collection. The cells were cultured as described in the supplemental methods.

All animal experiments were conducted in compliance with the national legislation on laboratory animal protection and with the approval of the Ethics Committee for Animal Research of Lund University. Male immunodeficient nude mice, NMRI-nu (Taconic) and BALB/c-nu (in house-bred), aged 6-8 wk, were inoculated in the right flank by a subcutaneous injection of $5-8 \times 10^{6}$ tumor cells in a $200-\mu \mathrm{L}$ cell suspension of a 1:1 mixture of medium with Matrigel (BD Biosciences). The tumors were allowed to develop for $6-8 \mathrm{wk}$. The animals were imaged under 2\%-3\% isoflurane anesthesia (Abbot Scandinavia). In Supplemental Table 1 , details are given on the different animal groups used in this study.

\section{CLI and SPECT Imaging}

CLI was performed using an in-house-built optical system based on a deep-cooled $\left(-85^{\circ} \mathrm{C}\right)$ charge-coupled device (iKon 934M; Andor) equipped with an objective lens (Xenon 25 mm, f/0.95; Schneider Kreuznach). The camera was placed on top of a light-tight cabinet. The exposure time was $10 \mathrm{~min}$, and no filter was used in front of the camera. Images were medianfiltered after background had been subtracted. The imaging system was intensity-calibrated as described in the supplemental methods.

Data were extracted from the CLI images by defining a region of interest (ROI) encircling the tumor. The average of the Cerenkov radiance within the ROI was calculated using values above a threshold at $50 \%$ of maximum intensity.

SPECT imaging was conducted using a preclinical SPECT/CT scanner (NanoSPECT/CT Plus; Bioscan) and the NSP-106 multipinhole mouse collimator. Energy windows of $20 \%$ were centered over the 56-, 113-, and 208-keV energy peaks. SPECT data were reconstructed using HiSPECT software (SciVis). Data were extracted from the SPECT image datasets by defining an ROI in 3 dimensions of the tumor in Matlab (version 8.1; The MathWorks Inc.). The ROI was assigned by encircling the tumor in each dimension. The ROI then was defined as the pixels holding a pixel count rate larger than $10 \%$ of the maximum count rate for the whole ROI. No anatomic registration with CT was performed in this study.

\section{Phantom Study}

To evaluate the ability of the CLI method to quantitatively measure the radioactivity, the Cerenkov radiance dependence of ${ }^{177} \mathrm{Lu}$ activity was investigated by CLI of a $24-w e l l$ plate (Thermo Scientific). The wells were filled with a mixture of water, intralipid (Fresenius Kabi), and radionuclide. Intralipid was used to mimic the scattering properties of tissue. Wells filled with ${ }^{18} \mathrm{~F}$, as a reference, were imaged for comparison of the CLI signal strength.

\section{Longitudinal Imaging of Tumor Uptake Using CLI and SPECT}

A prerequisite for individual dosimetry is the ability to image the animals undergoing treatment longitudinally. To investigate whether the ${ }^{177} \mathrm{Lu}$ activity in vivo could be imaged using CLI over several halflives, the animals were injected with ${ }^{177} \mathrm{Lu}-\mathrm{m} 11 \mathrm{~B} 6$ through the tail vein. CLI and SPECT images were acquired at 48, 72, 168, 264, and $336 \mathrm{~h}$ after injection. The CLI radiance and SPECT count rate were extracted from images of tumors and were plotted against time. Extracted data from 5 mice (2 BALB/c-nu and 3 NMRI-nu) were used to study the correlation of tumor uptake between CLI and SPECT.

The CLI and SPECT data analysis was performed by considering the whole tumor, in which the radionuclide distribution is assumed to be constant in time. In this case, the CLI radiance is taken to be directly proportional to the activity of the radionuclide. It is difficult, however, to explicitly retrieve the absolute activity from radiance measurements in vivo due to the large effects of light absorption and scattering. To convert the CLI radiance to units related to activity concentration, the CLI dataset was normalized using SPECT and CLI data at a single time point $(72 \mathrm{~h})$ and was scaled according to Equation 1.

$$
\mathrm{R}_{\mathrm{CLI}}(t)=\frac{\mathrm{R}_{\mathrm{SPECT}}(t=72 \mathrm{~h})}{\mathrm{L}_{\mathrm{CLI}}(t=72 \mathrm{~h})} \times \mathrm{L}_{\mathrm{CLI}}(t)
$$

Eq. 1

In Equation 1, $\mathrm{L}_{\mathrm{CLI}}(\mathrm{t})$ is the CLI radiance measured at different times after injection, whereas $R_{\text {SPECT }}(t)$ is the SPECT count rate. $R_{C L I}(t)$ is

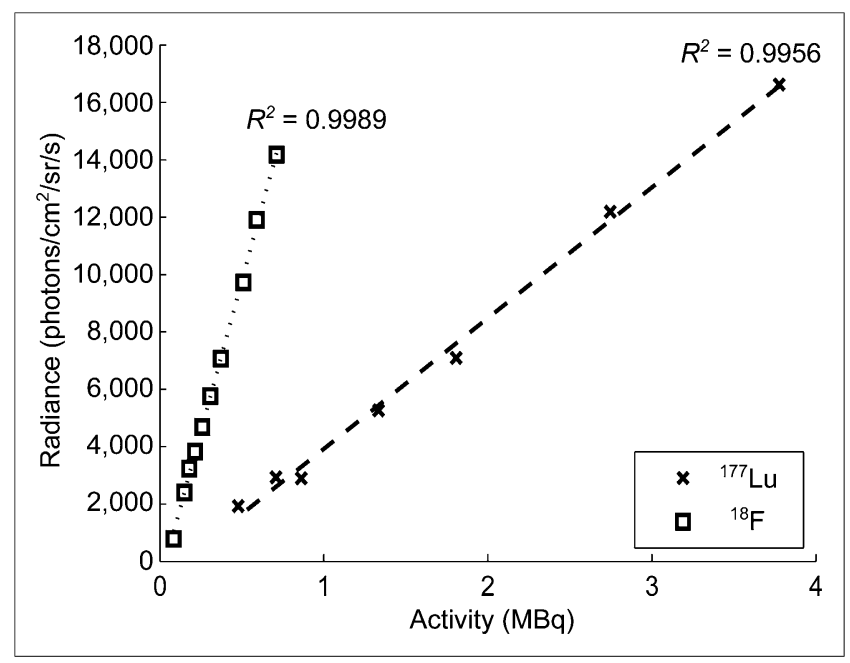

FIGURE 1. Well plate experiment to verify dependence of activity on $\mathrm{CLI}$ radiance in phantom solutions. Both ${ }^{18} \mathrm{~F}$ and ${ }^{177} \mathrm{Lu}$ show linear relationship between Cerenkov emission radiance and activity. 
the normalized Cerenkov radiance represented in units of counts per second.

\section{Quantification of Uptake and Tumor Response Monitoring Using CLI}

To investigate how the CLI radiance relates to the radionuclide specific uptake value, that is, percentage injected activity per gram (\%IA/g), 4 tumor-bearing $\mathrm{BALB} / \mathrm{c}$ nude mice were injected with ${ }^{177} \mathrm{Lu}-\mathrm{h} 11 \mathrm{~B} 6$ (20 MBq per animal). CLI images were acquired at 24, 48, 72, and $168 \mathrm{~h}$ after injection. The CLI radiance in vivo was extracted from the images by defining an ROI over the tumor and then averaging all pixel values within the ROI. The tumor weight was assessed at $168 \mathrm{~h}$, when the tumors were resected and weighed. The CLI radiance was then normalized to the tumor weight and was plotted against published specific uptake values from a similar biodistribution study (13). Briefly, in that study, 20 animals were injected with about $0.5 \mathrm{MBq}$ of ${ }^{177} \mathrm{Lu}$ and $20 \mu \mathrm{g}$ of labeled h11B6 per mouse. At 24 , 48,72 , and $168 \mathrm{~h}$ after injection, organs were resected, and the specific uptake value was assessed for each organ, with activity measured using a NaI(Tl) well counter (1480 Wallac Wizard; Perkin Elmer).

Quantification was done in the same way for 3 animals injected with $20 \mathrm{MBq}$ of ${ }^{177} \mathrm{Lu}-\mathrm{h} 11 \mathrm{~B} 6$, with the tumors being imaged at $168 \mathrm{~h}$ after injection and then resected. The tumor activity was measured using the well counter described above and then compared with the CLI radiance after both had been normalized to the tumor weight.

A pilot study was performed in which 4 animals undergoing radionuclide therapy with ${ }^{177} \mathrm{Lu}$-h11B6 were imaged during the first
$11 \mathrm{~d}$ after radiopharmaceutical administration. The tumor volume for each animal was estimated using caliper measurements during the treatment, which extended over several months. The tumor was measured in 2 dimensions, length (L) and width (W), and the volume was then calculated from $0.5 \times \mathrm{L} \times \mathrm{W} \times \mathrm{W}(14,15)$. The tissue density used was $1 \mathrm{~g} / \mathrm{cm}^{3}$ (16). CLI radiance was extracted from the tumor and was normalized to the respective tumor weight. The mean absorbed dose after injection at $t=0$ is given by

$$
\mathrm{D}_{r_{T}},\left(T_{D}\right)=\int_{0}^{T_{D}} \mathrm{~A}\left(r_{S}, t\right) \cdot \mathrm{S}\left(r_{T} \leftarrow r_{S}, t\right) d t,
$$

where $\mathrm{A}\left(r_{S}, t\right)$ is the activity at time $t$ in the source organ and $\mathrm{S}\left(r_{T} \leftarrow r_{S}, t\right)$ is the mean absorbed dose to the target tissue per amount of activity at the source organ at time $t$, defined by $\mathrm{S}\left(r_{T} \leftarrow r_{S}, t\right)=\frac{1}{m_{r} T} \sum_{i} \Delta_{i} \phi_{i}$ (17). Here, $\Delta_{i}$ is the mean energy of the $\mathrm{i}^{\text {th }}$ transition per nuclear transformation whereas $\phi_{i}$ is the absorbed fraction. Because of the range of the $\beta$ particles, the tumor tissue is the dominant source for activity contributing to the absorbed dose of the tumor, that is, $\phi_{i} \sim 1$. Ideally, the CLI radiance should be directly proportional to the activity (18). Based on this assumption, the CLI radiance could simply replace the activity within the MIRD formalism, that is, in Equation 2. Hence, the mean absorbed dose to the tumor $\left(D_{\text {tumor }}(t)\right)$ can be written as

$$
\mathrm{D}_{\text {tumor }}\left(T_{D}\right)=k \int_{0}^{T_{D}} \frac{\mathrm{L}_{\text {tumor }}(t)}{m_{\text {tumor }}(t)} \sum_{i} \Delta_{i} \phi_{i} d t,
$$

Eq. 3

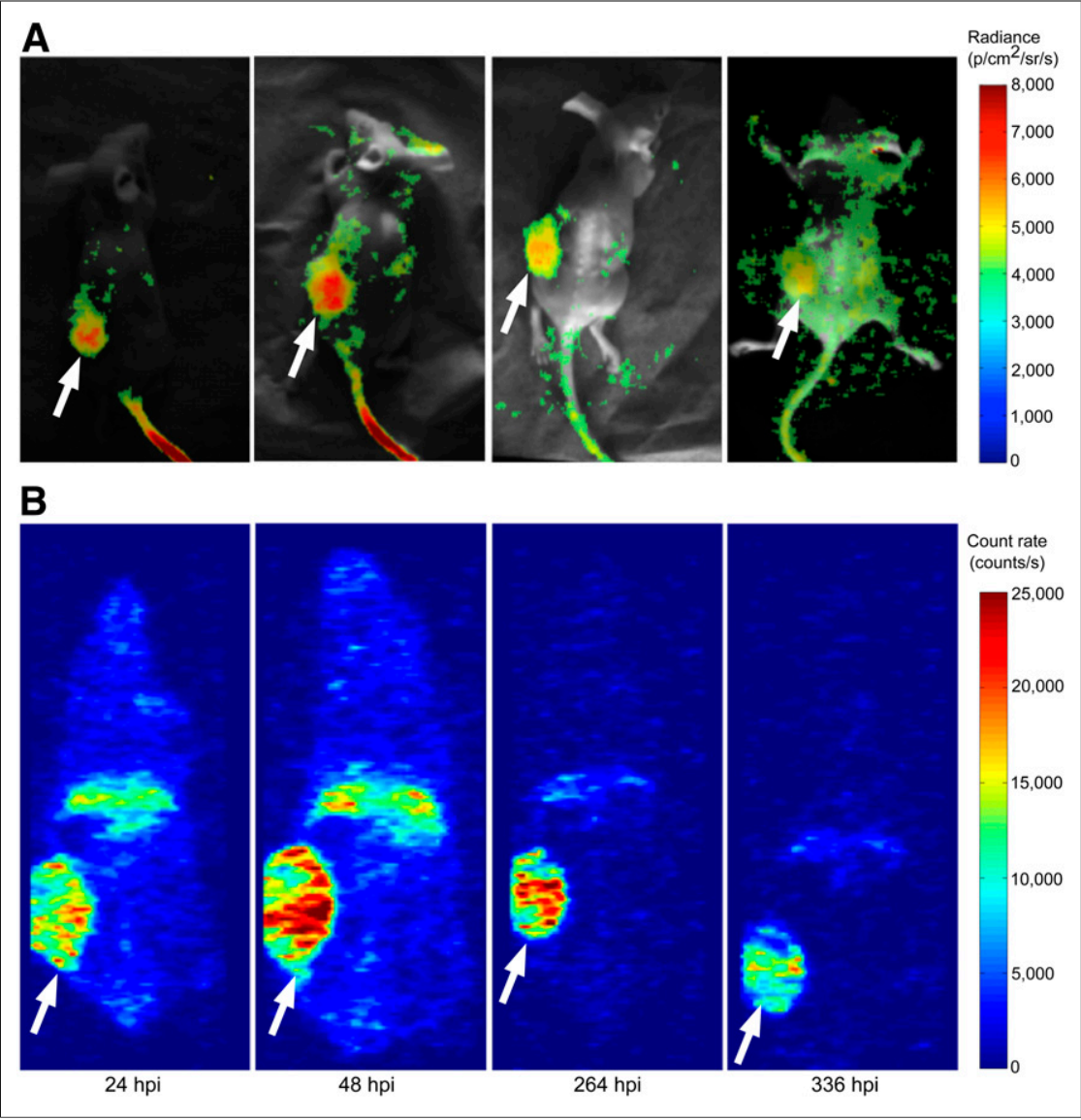

FIGURE 2. Longitudinal CLI images (A) and SPECT images (B) after administration of $20 \mathrm{MBq}$ of ${ }^{177} \mathrm{Lu}-\mathrm{m} 11 \mathrm{~B} 6$. Tumor (arrow) is well delineated in both CLI and SPECT images up to $336 \mathrm{~h}$ after injection (hpi). $\mathrm{sr}=$ steradian. where $k$ is a calibration constant and $\mathrm{L}_{\text {tumor }}(t)$ is the time-dependent CLI radiance from the tumor. Herein $\mathrm{D}_{\text {tumor }}$ is referred to as AUC. AUCs were integrated, using CLI radiance normalized to the tumor weight, for each animal at every imaging session at $24,48,72$, 168 , and $264 \mathrm{~h}$ after injection. The timedependent AUCs were then plotted against the respective tumor volume change to investigate whether CLI could reveal information about subject-specific treatment efficacy.

\section{RESULTS}

\section{Phantom Study}

The CLI radiance extracted from the images of the well plate is shown as a function of activity in Figure 1 for ${ }^{177} \mathrm{Lu}$ and ${ }^{18} \mathrm{~F}$. It is evident that ${ }^{18} \mathrm{~F}$ renders stronger CLI signal than ${ }^{177} \mathrm{Lu}$ for a given activity. The main contributor to this result is that the kinetic energy of the $\beta$ particles from ${ }^{18} \mathrm{~F}$ is higher than that from ${ }^{177} \mathrm{Lu}$ (maximum energy, 0.635 and $0.497 \mathrm{MeV}$, respectively). Hence, there are more $\beta$ particles satisfying the Cerenkov threshold condition, leading to more Cerenkov photon emissions (18).

\section{Longitudinal Imaging of Tumor Uptake Using CLI and SPECT}

Examples of CLI and SPECT images acquired from a single animal at different times after injection are shown in Figure 2. The increasing amount of activity in the 
tumor resulted in an increasing CLI radiance and SPECT count rate, indicating that both methods can be used to follow the specific uptake of the radiolabeled antibody. ${ }^{177} \mathrm{Lu}$ can be visualized up to $2 \mathrm{wk}$ after an injection of approximately $20 \mathrm{MBq}$ per animal. The CLI signal from the tail is most likely due to a combined effect of extravasation at injection and superficial vasculature. The tail is not seen in the SPECT images since it is outside the field of view.

CLI radiance and SPECT count rate for the tumor were extracted from the images and, in Figures 3A and 3B, are plotted for 2 animals as a function of time after injection. CLI and SPECT data then were used to fit a 2-compartment model (19), that is, a double exponential function (Matlab, version 8.1) for the tumor biokinetics, which is shown in Figures 3A and 3B, as guidance for the eye. The CLI and SPECT kinetics agreed well, with some small deviations that most likely were due to differences in exact positioning of the animals but could also be attributed to changes in tumor volume, since normalization to the tumor weight was not performed here. When the curves were integrated, the values showed a deviation of only $4 \%$ and $6 \%$ between CLI and SPECT. This deviation is small compared with the total integrated values for SPECT of $3.5 \times 10^{6}$ and $2.8 \times 10^{6}$ counts, which is a difference of $25 \%$ between the 2 animals. When the CLI radiance and SPECT count rates were compared for all 5 animals (Fig. 3C), the correlation was strong. This finding is in agreement with other studies in which PET images were compared with CLI (7-9). The CLI radiance scaled in units of SPECT count rate, calculated using Equation 1, is shown as a function of the SPECT count rate in Figure 3D.

\section{Quantification of Uptake Using CLI}

Representative CLI images of a single animal injected with $20 \mathrm{MBq}$ of ${ }^{177} \mathrm{Lu}-\mathrm{h} 11 \mathrm{~B} 6$ are shown in Figure 4A. CLI radiance was extracted by defining an ROI in the images of the tumor. The CLI radiance was then normalized to the tumor weight $(\% \mathrm{IA} / \mathrm{g})$, to allow comparison with the previously obtained specific uptake (13). The normalized CLI radiance is compared with the specific uptake value for the tumor in Figure 4B. In Figure 4C, the extracted CLI radiance and the \%IA/g are from the same animals. A linear trend was seen between the normalized CLI radiance and the specific uptake value. According to the results in Figure 4, CLI radiance and the specific uptake value, assessed using ex vivo measurements, correlated well. This finding agrees with reported results for PET compared with CLI (7-9). However, the scenario during radionuclide therapy imaging was slightly different from PET, since tumor weight changes drastically during the weeks when imaging is performed. In this study, tumor weight was assessed from the tumor volume, calculated using caliper measurements, which have large variability (20), partly explaining the relatively large error bars in Figure 4B.

\section{Tumor Response Monitoring Using CLI}

In Figures $5 \mathrm{~A}$ to $5 \mathrm{D}$, the relative tumor volume is plotted against AUC, calculated at different times after injection, for each animal together with an exponential fit of the data. In some cases the tumor volume increased from day 0 to day 2 after injection (Fig. 5C), leading to values larger than unity. The AUCs were evaluated at the time of each imaging session. In Figures $5 \mathrm{~A}$ to $5 \mathrm{D}$, tumor volume is seen to decrease exponentially with increasing AUC.

\section{DISCUSSION}

In this work, the feasibility of CLI as a tool for monitoring individual biokinetics during preclinical radionuclide therapy was investigated. Several aspects were studied. First, the correlation between SPECT images and CLI of the tumor showed that it is possible to perform CLI imaging that correlates with SPECT up to 2 wk after a single injection of $20 \mathrm{MBq}$ of ${ }^{177} \mathrm{Lu}-11 \mathrm{~B} 6$. Second, the correlation between the standardized uptake value (\%IA/g) and CLI, normalized to the tumor weight, showed a linear correlation for the tumor. Third, the tumor volume change during the course of treatment showed an exponential decrease when plotted against the integrated CLI signal as a function of time, that is, the AUC. It is well established that tumor volume decreases exponentially in response to increasing absorbed dose (3). The relationship in Equations 2 and 3 shows that the CLI-based AUC is directly proportional to the absorbed dose. The observed correlation 


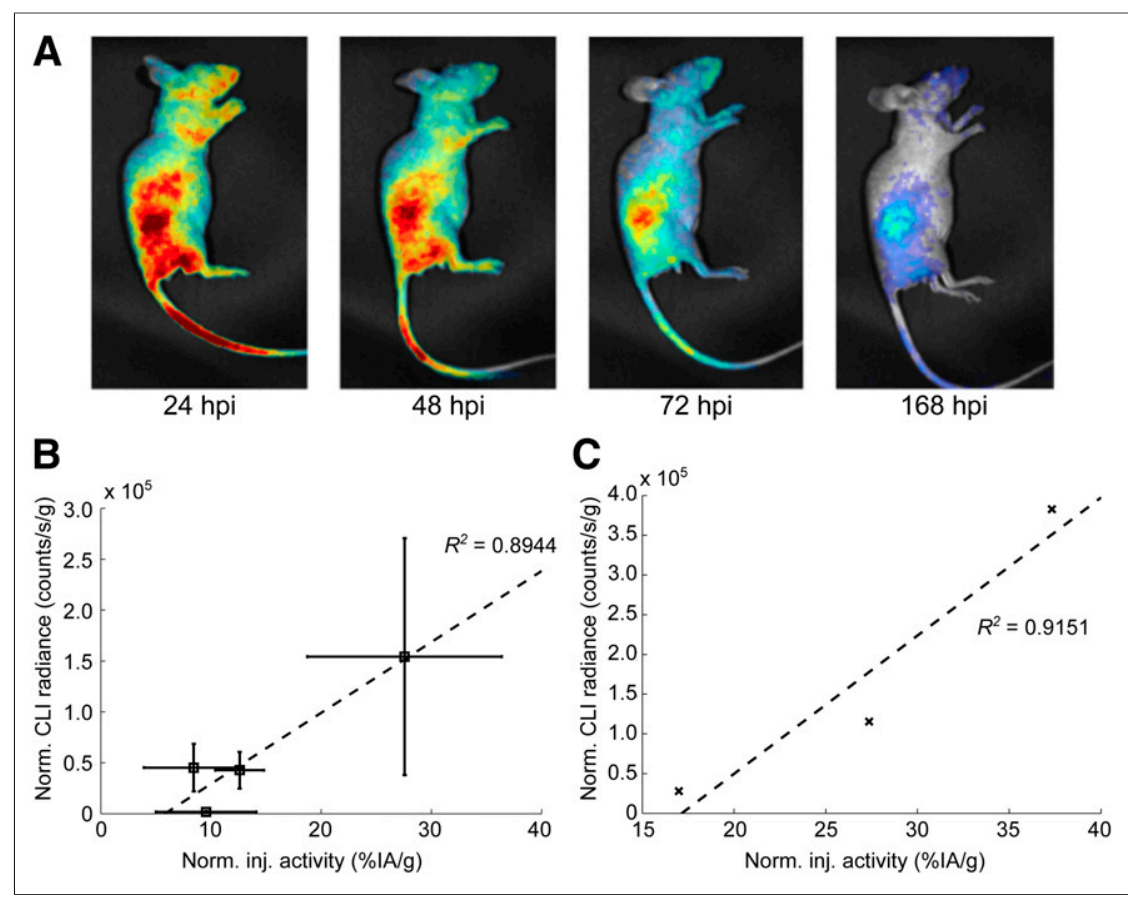

FIGURE 4. Comparison between $\mathrm{CLI}$ and specific uptake value $(\% \mathrm{IA} / \mathrm{g})$. (A) Longitudinal $\mathrm{CL}$ images of 1 animal after administration of $20 \mathrm{MBq}$ of ${ }^{177} \mathrm{Lu}$-h11B6. (B) CLI radiance, normalized to tumor weight, compared with biodistribution data compiled from previously published study (13). Vertical error bars are SD of CLI radiance $(n=4)$, whereas horizontal error bars are SD from previously reported biodistribution study $(n=20)$. (C) CLI radiance shown as function of specific uptake $(\% \mathrm{IA} / \mathrm{g})$ from excised tumor for 3 mice at $168 \mathrm{~h}$ after injection.
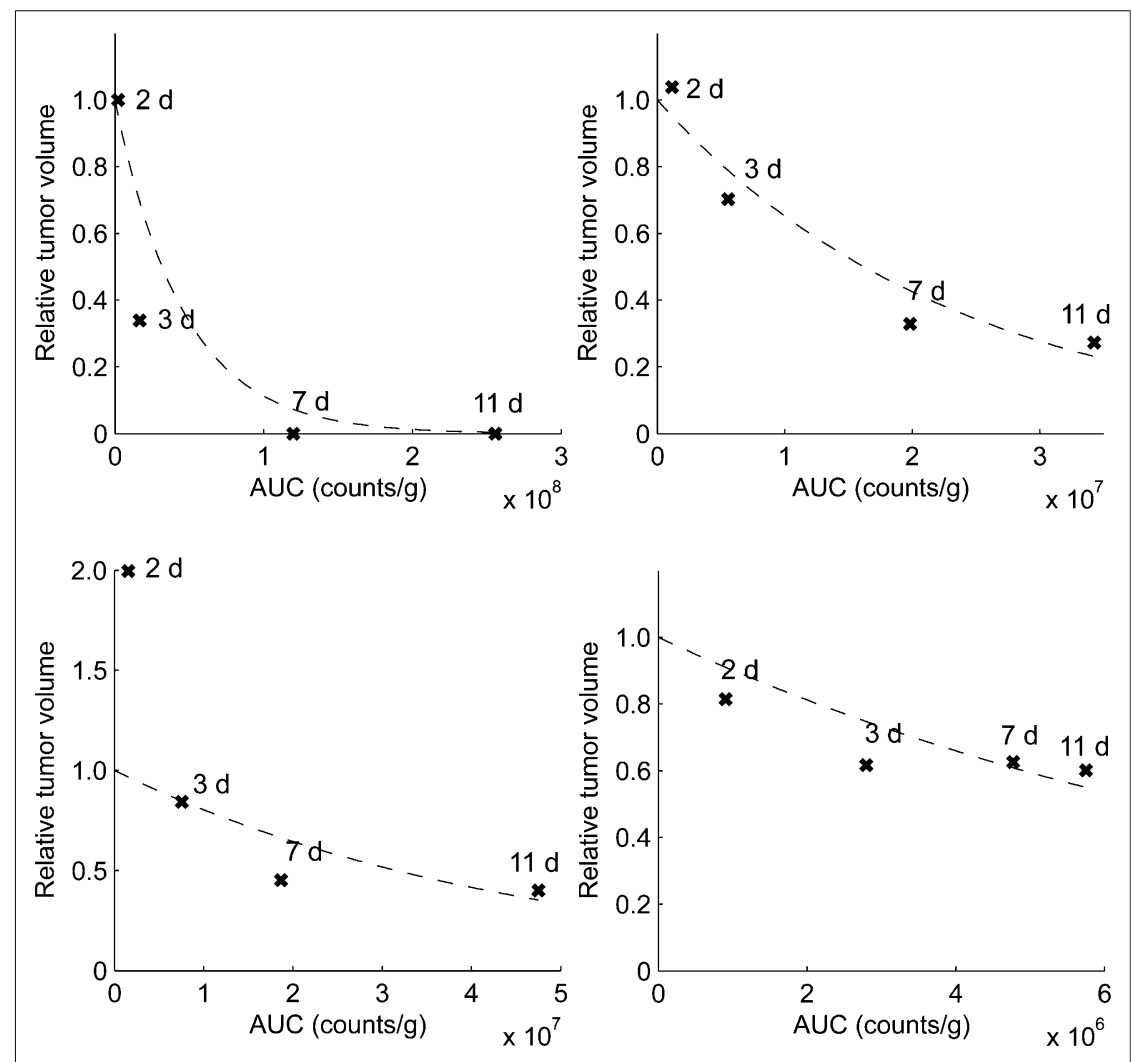

FIGURE 5. Tumor response monitoring using CLI. Each graph is relative tumor volume for 1 of 4 animals during first $11 \mathrm{~d}$ plotted against AUC, calculated at time of each imaging session. between tumor volume reduction and AUC is thus encouraging, indicating the usefulness of CLI. Although no calculation based on the AUC of the absorbed dose (Gy) was performed, the method shows promise in that it elucidates differences in the treatment effect between animals.

Dosimetry in radionuclide therapy is essential to enable a correlation with biologic response. Many new therapeutic radiopharmaceuticals are investigated in tumor-bearing rodents before translation to clinical trials. In animals, biologic parameters such as physiology may differ between individuals. Thus, methods for longitudinal studies, in which the biokinetics of each individual animal within a study are monitored preferably in relation to observed biologic effects, are warranted.

In this study, we have shown that the easier and less time-consuming method relying on CLI can be used to follow tumor biokinetics. The results show that even a weak Cerenkov emitter such as ${ }^{177} \mathrm{Lu}$ can be imaged during $2 \mathrm{wk}$ after injection, given sufficient tumor retention, and that the correlation with in vivo SPECT quantification or with ex vivo specific uptake values is good. However, CLI reports the data in units of radiance, which are not relevant for radionuclide therapy dosimetry. In this study, we propose a simple normalization scheme in which the SPECT or specific uptake value at a single time point is used to normalize the CLI signal. In this way, CLI accounts for the longitudinal changes, whereas SPECT or specific uptake value imposes accurate units on the data. This limits the need for time-consuming longitudinal SPECT imaging and relies instead on the CLI technique to follow the radiopharmaceutical kinetics in the tumor. This approach is used in clinical therapy studies in which several planar scintillation camera images combined with a single SPECT image for activity calibration have been used (21). The method proposed here could be used in the same way with CLI for planar imaging and SPECT for calibration. However, because there is not a perfect match between CLI and SPECT at later time points, as discussed in relation to Figures $3 \mathrm{~A}$ and $3 \mathrm{~B}$, a small uncertainty in the proposed calibration method could be introduced.

CLI is an imaging modality that relies on detection of optical photons. Most of the Cerenkov emission spectrum is in the visible range, where blood is the most prominent absorber (22). Hence, it is expected that the blood volume in tissue affects CLI radiance. 
This fact can explain the large SD in Figure 4B and the variability in Figures 5A-5D. A potential weakness of the proposed CLI method is if the blood volume changes dramatically during treatment. A lower blood volume will result in a stronger Cerenkov signal. On the other hand, the effect seems to be moderate since the CLI and SPECT correlation is strong, as seen in Figures 3C and 3D. Another analogous effect is the effective depth of the tumor that will be affected when the tumor volume is reduced. A greater depth will render a longer propagation distance for the Cerenkov emission; hence, the CLI signal will decrease. It is important to know how large these effects are in absolute values, and this is being investigated within our current work. Approaches based on Cerenkov luminescence tomography can, however, be used to assess the radioactivity quantitatively within the tissue based on a model-based approach (23). The fact that these methods require a priori data, for example, retrieval of the body shape and knowledge of the optical absorption and scattering, reduces the high-throughput capability.

In this study, the integrated CLI signal as a function of time was used as a dose metric, referred to as AUC. Since the AUC is calculated from the CLI signal, the units are in radiance, which has no dosimetric meaning. However, Figures 3 and 4 show that the CLI radiance was directly proportional to SPECT or to specific uptake value, respectively. Hence, using SPECT at a single time point in addition to CLI measurements at several time points potentially could render a calibrated AUC that is useful for calculating accumulated activity. For this study, however, the AUC in CLI units was used to investigate how the AUC from CLI relates to the tumor volume. The normalization schemes presented in Equation 1 can serve as a first approach to convert the CLI radiance to a quantity, that is, specific uptake $(\% \mathrm{IA} / \mathrm{g})$, that can be applied directly for absorbed dose calculation. The ultimate goal of using CLI as a high-throughput complement to SPECT during radionuclide therapy is to assess the absorbed dose from the time-activity curves. The method presented here shows promise in that the tumor volume reduction correlates with the AUC calculated from the CLI images. However, to assess the accuracy of the proposed method, a larger study with more treatment subjects should be performed.

\section{CONCLUSION}

The results presented here suggest that CLI can be used well as a complementary imaging technique that allows for longitudinal monitoring of ${ }^{177} \mathrm{Lu}$-based radionuclide therapy. We suggest that CLI can be used to more accurately follow the individual tumor biokinetics intratherapeutically in preclinical radionuclide therapy. This approach could be a valuable methodology for high-throughput individual monitoring of radionuclide therapy.

\section{DISCLOSURE}

The costs of publication of this article were defrayed in part by the payment of page charges. Therefore, and solely to indicate this fact, this article is hereby marked "advertisement" in accordance with 18 USC section 1734. This study was supported by The Research School in Pharmaceutical Sciences (FLÄK, Lund University), the Swedish Cancer Foundation, the Swedish Science Council, Mrs. Berta Kamprad's Foundation, Gunnar Nilsson's Foundation, Percy Falk's Foundation, and government funding of clinical research within the NHS (National Health Service) of Lund University, Sweden (ALF). Dr. Strand is a shareholder of DiaProst (Lund, Sweden), which holds a patent for human kallikrein-related peptidase 2 targeting. Dr. Tran holds stock options in DiaProst. DiaProst has not financed any part of this study.
Lund University Bioimaging Center (LBIC) provided experimental resources. No other potential conflict of interest relevant to this article was reported.

\section{ACKNOWLEDGMENTS}

We thank Anna Åkesson and Jörgen Elgqvist at the Department of Medical Radiation Physics and Gustav Grafström at LBIC for technical assistance.

\section{REFERENCES}

1. Larsson E, Ljungberg M, Martensson L, et al. Use of Monte Carlo simulations with a realistic rat phantom for examining the correlation between hematopoietic system response and red marrow absorbed dose in brown Norway rats undergoing radionuclide therapy with Lu-177- and Y-90-BR96 mAbs. Med Phys. 2012;39:4434-4443.

2. Larsson E, Ljungberg M, Strand S-E, Jönsson B-A. Monte Carlo calculations of absorbed doses in tumours using a modified MOBY mouse phantom for pre-clinical dosimetry studies. Acta Oncol. 2011;50:973-980.

3. Hindorf C, Linden O, Stenberg L, Tennvall J, Strand SE. Change in tumorabsorbed dose due to decrease in mass during fractionated radioimmunotherapy in lymphoma patients. Clin Cancer Res. 2003;9(suppl):4003S-4006S.

4. Robertson R, Germanos MS, Li C, et al. Optical imaging of Cerenkov light generation from positron-emitting radiotracers. Phys Med Biol. 2009;54:N355-N365.

5. Liu H, Ren G, Miao Z, et al. Molecular optical imaging with radioactive probes. PLoS ONE. 2010;5:e9470.

6. Thorek DL, Robertson R, Bacchus W, et al. Cerenkov imaging: a new modality for molecular imaging. Am J Nucl Med Mol Imaging. 2012;2:163-173.

7. Ruggiero A, Holland JP, Lewis JS, Grimm J. Cerenkov luminescence imaging of medical isotopes. J Nucl Med. 2010;51:1123-1130.

8. Holland JP, Normand G, Ruggiero A, Lewis JS, Grimm J. Intraoperative imaging of positron emission tomographic radiotracers using Cerenkov luminescence emissions. Mol Imaging. 2011;10:177-186.

9. Xu Y, Chang E, Liu H, Jiang H, Gambhir SS, Cheng Z. Proof-of-concept study of monitoring cancer drug therapy with Cerenkov luminescence imaging. $J$ Nucl Med. 2012;53:312-317.

10. Price EW, Zeglis BM, Cawthray JF, et al. $\mathrm{H}_{4}$ octapa-trastuzumab: versatile acyclic chelate system for ${ }^{111} \mathrm{In}$ and ${ }^{177} \mathrm{Lu}$ imaging and therapy. J Am Chem Soc. 2013; 135:12707-12721.

11. Balkin ER, Kenoyer A, Orozco JJ, et al. In vivo localization of ${ }^{90} \mathrm{Y}$ and ${ }^{177} \mathrm{Lu}$ radioimmunoconjugates using Cerenkov luminescence imaging in a disseminated murine leukemia model. Cancer Res. 2014;74:5846-5854.

12. Nuclear Decay Data in the MIRD Format. Upton, NY: National Nuclear Data Center, Brookhaven National Laboratory; 2014.

13. Vilhelmsson Timmermand O, Larsson E, Strand S-E, Tran T. In vivo evaluation of ${ }^{177} \mathrm{Lu}$-labeled h11B6, a humanized antibody for hK2 targeting in prostate cancer [abstract]. J Nucl Med. 2014;55(suppl): 1485.

14. Euhus DM, Hudd C, LaRegina MC, Johnson FE. Tumor measurement in the nude mouse. J Surg Oncol. 1986;31:229-234.

15. Tomayko MM, Reynolds CP. Determination of subcutaneous tumor size in athymic (nude) mice. Cancer Chemother Pharmacol. 1989;24:148-154.

16. ICRP Composition of Soft Tissue. Gaithersburg, MD: National Institute of Standards and Technology; 2014.

17. Bolch WE, Eckerman KF, Sgouros G, Thomas SR. MIRD pamphlet no. 21: a generalized schema for radiopharmaceutical dosimetry-standardization of nomenclature. J Nucl Med. 2009;50:477-484.

18. Beattie BJ, Thorek DLJ, Schmidtlein CR, Pentlow KS, Humm JL, Hielscher AH. Quantitative modeling of Cerenkov light production efficiency from medical radionuclides. PLOS ONE. 2012;7:e31402.

19. Vicini P, Brill AB, Stabin MG, Rescigno A. Kinetic modeling in support of radionuclide dose assessment. Semin Nucl Med. 2008;38:335-346.

20. Ayers GD, McKinley ET, Zhao P, et al. Volume of preclinical xenograft tumors is more accurately assessed by ultrasound imaging than manual caliper measurements. J Ultrasound Med. 2010;29:891-901.

21. Sjögreen K, Ljungberg M, Wingardh K, Minarik D, Strand SE. The LundADose method for planar image activity quantification and absorbed-dose assessment in radionuclide therapy. Cancer Biother Radiopharm. 2005;20:92-97.

22. Axelsson J, Glaser AK, Gladstone DJ, Pogue BW. Quantitative Cherenkov emission spectroscopy for tissue oxygenation assessment. Opt Express. 2012;20:5133-5142.

23. Li C, Mitchell GS, Cherry SR. Cerenkov luminescence tomography for small animal imaging. Opt Lett. 2010;35:1109-1111. 